\title{
Optical Coherence Tomography as a Useful Objective Modality in Diagnosing and Treating Corticosteroid Induced Secondary Pseudotumor Cerebri Syndrome in a Child with Severe Eczema \\ SA Lalchan ${ }^{1}$, N Hallai $^{2}$, VRS Singh ${ }^{2}$
}

\begin{abstract}
Secondary pseudotumor cerebri syndrome following corticosteroid withdrawal is well a documented entity. Timely diagnosis is critical to preventing blindness. It can be particularly challenging to diagnose in very young children as they may not be able to verbalise the symptoms of headaches and visual loss. Additionally, there can be diagnostic uncertainty if there is subtle papilledema or clinical examination is difficult. Optical coherence tomography can be a very useful tool to both diagnose and monitor papilledema as demonstrated in this 4 year old child following steroid withdrawal for severe eczema. The authors also aim to highlight the importance of the multidisciplinary team in successful management of these challenging cases.
\end{abstract}

Keywords: Eczema, papilledema secondary pseudotumor cerebri

From: ${ }^{1}$ LILY-The Eye Specialist Ltd, Chaguanas, Trinidad and Tobago, ${ }^{2}$ Eric Williams Medical Sciences Complex, Champ Fleurs, Trinidad and Tobago.

Correspondence: Ms S Lalchan, \#8 Endeavour Road, Chaguanas, Trinidad and Tobago West Indies. Email: mslalchan@ gmail.com 


\section{Corticosteroid Induced Secondary Pseudotumor Cerebri Syndrome}

\section{INTRODUCTION}

Secondary pseudotumor cerebri syndrome is well documented with risk factors inclusive of tetracyclines, retinoids, corticosteroid, growth hormone withdrawal and renal diseases (1-5). The revised criteria by Friedman et al aims to improve the diagnostic sensitivity and specificity in both adults and paediatric patients $(1,6)$. Early diagnosis is pivotal as the rates for permanent visual impairment varies from 10-40\% [Table 1] $(7,8)$. This can be challenging in very young patients as they may not be able to verbalise the symptoms of headaches and visual loss. Additionally, there can be diagnostic uncertainty if there is subtle papilledema or clinical examination is difficult; modern ophthalmic imaging can be a useful objective tool in these patients.

The authors would like to present a $4 \mathrm{yr}$ old boy with severe eczema treated with intramuscular triamcinolone injections for over $1 \mathrm{yr}$, who was diagnosed with secondary pseudotumor cerebri syndrome, failure to thrive and uncontrolled eczema. Optical coherence tomography was used to successfully guide the diagnostic and therapeutic challenges of this clinical scenario $(9,10)$.

\section{CASE REPORT}

A $4 \mathrm{yr}$ old boy was referred by the optician for headaches over the past month. He was unfortunately treated by a general practitioner for severe eczema with weekly intramuscular triamcinolone $40 \mathrm{mg}$ for one month, followed by monthly intramuscular triamcinolone $40 \mathrm{mg}$ for a subsequent fourteen months (Fig 1). He was also on oral anti-histamine and topical antifungal creams. The last injection was two months prior to the presentation. He was below the fifth centile for his age: height $11.3 \mathrm{~kg}$ and weight $95 \mathrm{~cm}$.

Ophthalmic examination was as follows: Snellen visual acuity 6.75 both eyes (BE), refraction +0.25DS BE; intraocular pressures were $16 \mathrm{mmHg}$ Goldmann's tonometry $(\mathrm{BE})$; 
dilated fundoscopy, fundus photography and optical coherence imaging (OCT) showed elevated nerve fibre layer confirmed Frisén papilledema grade 1 in the right eye and grade 2 in the left eye (Fig 2).

The child was referred to the paediatric accident and emergency and admitted. He was subsequently managed by a multidisciplinary team inclusive of ophthalmologist, paediatrician, dermatologist and neurologist. Secondary intracranial hypertension was confirmed with a lumbar puncture, normal CSF cytology; MRI (magnetic resonance imaging) of the head showed distension of optic nerve sheaths with flattened macula. He was successfully managed with oral acetazolamide (Fig 3).

The severe, rebound erythrodermic eczema was treated with oral azathioprine 2$3 \mathrm{mg} / \mathrm{kg}$, intensive moisturisers, dry wrapping and topical pimecrolimus to the face in a subsequent prolonged inpatient stay. There was concern by the ophthalmologist that further topical/oral corticosteroids may exacerbate the secondary pseudotumor cerebri. A report was sent to the local regulatory body as per good clinical practice.

The child was reviewed monthly by the ophthalmologist over the past year. OCT showed clear consistent response to the acetazolamide (Fig 4). The headaches have resolved, there is charted growth and control of the eczema (Fig 1) with a significant improvement in the child's quality of life.

\section{DISCUSSION}

Idiopathic intracranial hypertension or pseudotumor cerebri is an entity of ill-defined etiology. It is characterised by raised intracranial hypertension, normal CSF biochemistry and normal neuroimaging (Table 1). Secondary pseudotumor cerebri (SPC) is a subset within which specific medical conditions and pharmacological associations have been documented. Corticosteroid withdrawal is a well-known feature (3-5). Interestingly, adrenal hormones are 


\section{Corticosteroid Induced Secondary Pseudotumor Cerebri Syndrome}

important for activation and trophic effects on cell survival, differentiation, maturation and synaptogenesis of the central nervous system. It is therefore not surprising that various exogenous and endogenous disorders of the hypothalamic-pituitary-axis lead to SPC (11).

The commonest presenting feature is headaches; $90 \%$ presents with headaches, $62 \%$ with transient visual loss and 52\% with transient tinnitus. In children the most consistent features are headaches and visual loss as it is difficult for children to verbalise the symptoms (12). Notably, patients with pseudotumor cerebri have several deficits in cognitive function such as patients, including memory, executive function, visual-spatial processing, attention, motor skills, working memory, and processing speed; these can persist up to three months despite successful reduction in the intracranial pressures (12).

Systemic features in children include diplopia (abducens palsy), ataxia, nuchal rigidity and facial palsy. Ophthalmic features may include papilledema (Frisén grading Table 2); macular edema, retinal haemorrhages, subretinal fluid and rarely subretinal neovascularisation. The feared complication is anterograde degeneration of the nerve fibre layer (optic nerve atrophy) with permanent visual loss.

Ophthalmic investigations include fundus photography, OCT and transorbital ultrasound. Fundus photography is a useful tool even at this age with the child's co-operation. The features on OCT include swelling of the nerve fibre layer at the optic nerve (papilledema) and retinal ganglion cell-inner plexiform layer complex (GCL-IPL) thickness (13). The presence of the latter features is important as the reversibility may indicate a better visual recovery and prognosis for the patient. Of course, CSF spinal taps and neuroimaging are part of the armamentarium and included here for completeness.

The treatment for SPC is cessation of instigating factors and control of the ICP. The goals are to reduce the headaches with improved quality of life and prevent visual loss. These 
can be achieved medically and/or surgically $(14,15)$. Medical treatment largely includes acetazolamide.

Surgical interventions are considered if medical treatment has failed in the presence of vision threatening optic neuropathy (16). Options include CSF deviation (ventriculoperitoneal shunts/ lumboperitoneal shunts); optic nerve sheath fenestration which will reduce the pressures on the optic nerve head but does not necessarily alleviate headaches; endovascular venous stenting in the presence of venous stenosis is also an alternative.

The treatment tier is guided by the success in achieving the aims outlined above. The ophthalmologist has an important role in assessing the clinical response of visual recovery and resolution of the optic nerve and retinal features. OCT of the RNFL serves as an objective response as seen in our patient. In chronic papilledema with macular changes, resolution may improve vision and its possible reversibility is an important prognostic feature. It is important to note that a reduction in OCT optic nerve swelling in chronic cases may be unable to differentiated RNFL due to reduction in swelling or atrophy of the cells as it measures structure; other functional assessments such as vision, pupillary response and Ishihara plates remain important clinical criteria. Lastly, in challenging cases the importance of a multidisciplinary team and early referral for secondary/tertiary care can reduce both morbidity and mortality. The child is well adjusted in school and has attended his first school outing. The team could not be more delighted 


\section{REFERENCES}

1. Paley GL, Sheldon CA, Burrows EK, Chilutti MR, Liu GT, McCormack SE. Overweight and obesity in pediatric secondary pseudotumor cerebri syndrome. Am J Ophthalmol 2015; 159: 344-52.

2. Ray WZ, Lee A, Blackburn SL, Lueder GT, Leonard JR. Pseudotumor cerebri following tapered corticosteroid treatment in an 8-month-old infant. J Neurosurg Pediatr. 2008; 1: 88-90.

3. Lorrot M, Bader-Meunier B, Sébire G, Dommergues JP. Benign intracranial hypertension: an unrecognized complication of corticosteroid therapy.Arch Pediatr. 1999 Jan; 6(1):40-2.

4. Liu GT, Kay MD, Bienfang DC, Schatz NJ. Pseudotumor cerebri associated with corticosteroid withdrawal in inflammatory bowel disease. Am J Ophthalmol. 1994 Mar 15; 117(3):352-7.

5. Victorio MC, Rothner AD. Diagnosis and treatment of idiopathic intracranial hypertension (IIH) in children and adolescents. Curr Neurol Neurosci Rep. 2013 Mar; 13(3):336.

6. Friedman $\mathrm{DI}^{1}$, Liu GT, Digre KB. Revised diagnostic criteria for the pseudotumor cerebri syndrome in adults and children. Neurology. 2013 Sep 24;81(13):1159-65.

7. Soiberman U, Stolovitch C, Balcer LJ, Regenbogen M, Constantini S, Kesler A. Idiopathic intracranial hypertension in children: visual outcome and risk of recurrence. Childs Nerv Syst. 2011; 27:1913-8.

8. Yri HM, Wegener M, Sander B, Jensen R. Idiopathic intracranial hypertension is not benign: a long-term outcome study. J Neurol. 2012 May; 259(5):886-94.

9. Scott CJ, Kardon RH, Lee AG, Frisén L, Wall M. Diagnosis and grading of papilledema in patients with raised intracranial pressure using optical coherence 
tomography vs clinical expert assessment using a clinical staging scale. Arch Ophthalmol. 2010; 128(6):705-11.

10. OCT Sub-Study Committee for NORDIC Idiopathic Intracranial Hypertension Study Group, Auinger P, Durbin M, Feldon S, Garvin M, Kardon R, Keltner J, Kupersmith MJ, Sibony P, Plumb K, Wang JK, Werner JS. Baseline OCT measurements in the idiopathic intracranial hypertension treatment trial, part II: correlations and relationship to clinical features. Invest Ophthalmol Vis Sci 201; 55: 8173-9.

11. Salpietro V, Polizzi A, Di Rosa G, Romeo AC, Dipasquale V, Morabito P, Chirico V, Arrigo T, Ruggieri M. Adrenal disorders and the paediatric brain: pathophysiological considerations and clinical implications. Int J Endocrinol. 2014; 2014:282489.

12. Julayanont P, Karukote A, Ruthirago D, Panikkath D, Panikkath R. Idiopathic intracranial hypertension: ongoing clinical challenges and future prospects. J Pain Res. 2016 Feb 19; 9:87-99.

13. Chen JJ, Thurtell MJ, Longmuir RA, Garvin MK, Wang JK, Wall M, Kardon RH. Causes and Prognosis of Visual Acuity Loss at the Time of Initial Presentation in Idiopathic Intracranial Hypertension. Invest Ophthalmol Vis Sci. 2015 Jun; 56(6):3850-9.

14. Wall M, McDermott MP, Kieburtz KD, Corbett JJ, Feldon SE, Friedman DI, Katz DM, Keltner JL, Schron EB, Kupersmith MJ. Effect of acetazolamide on visual function in patients with idiopathic intracranial hypertension and mild visual loss: the idiopathic intracranial hyperteNORDIC Idiopathic Intracranial Hypertension Study Group Writing Committee, JAMA. 2014 Apr 23-30;311(16):1641-51.

15. Cochrane Database Syst Rev. 2015 Aug 7;8:CD003434. 


\section{Corticosteroid Induced Secondary Pseudotumor Cerebri Syndrome}

16. Per H, Canpolat M, Gümüş H, Poyrazoğlu HG, Yıkılmaz A, Karaküçük S, Doğan H, Kumandaş S. Clinical spectrum of the pseudotumor cerebri in children: etiological, clinical features, treatment and prognosis. Brain Dev. 2013 Jun;35(6):561-8. 
Table $1 \quad$ Diagnostic criteria for pseudotumor cerebri syndrome ${ }^{6}$

1 Required for diagnosis of pseudotumor cerebri syndrome ${ }^{a}$

A. Papilledema

B. Normal neurologic examination except for cranial nerve abnormalities

C. Neuroimaging: Normal brain parenchyma without evidence of hydrocephalus, mass, or structural lesion and no abnormal meningeal enhancement on MRI, with and without gadolinium, for typical patients (female and obese), and MRI, with and without gadolinium, and magnetic resonance venography for other; if MRI is unavailable or contraindicates, contrast-enhanced CT may be used

D. Normal CSF composition

E. Elevated lumbar puncture opening pressure $(\geq 250 \mathrm{~mm}$ CSF in adults and $\geq 280 \mathrm{~mm}$ CSF in children [250mm CSF if the child is not sedated and not obese] in a properly performed lumber puncture

\section{Diagnosis of pseudotumor cerebri syndrome without papilledema}

In the absence of papilledema, a diagnosis of pseudotumor cerebri syndrome can be made if B-E from above are satisfied, and in addition the patient has a unilateral or bilateral abducens of nerve palsy

In the absence of papilledema or sixth nerve palsy, a diagnosis of pseudotumor cerebri syndrome can be suggested but not made if B_E from above are satisfied, and in addition at least 3 of the following neuroimaging criteria are satisfied

i. Empty Sella

ii. Flattening of the posterior aspect of the globe

iii. Distention of the perioptic subarachnoid space with or without a tortuous optic nerve

iv. Transverse venous sinus stenosis

${ }^{\mathrm{a}}$ A diagnosis of pseudotumor cerebri syndrome is definite if the patient fulfils criteria A-E. The diagnosis is considered probable if criteria A-D are met but the measured CSF pressure is lower than specified for a definite diagnosis. 
Modified Frisén Scale

\begin{tabular}{ll}
\hline Papilledema Grade Criteria \\
\hline 0 Promineer
\end{tabular}

o Normal Optic Disc

Prominence of the retinal nerve fibre layer at the nasal, superior, and inferior poles in inverse proportion to disc diameter

Radial nerve fibre layer striations, without tortuosity

1 Minimal Degree of Edema C-shaped halo that is subtle and greyish with a temporal gap; obscures underlying retinal details ${ }^{\mathrm{a}}$

Disruption of normal radial nerve fibre layer arrangement striations

Temporal disc margin normal

2 Low Degree of Edema Circumferential halo ${ }^{a}$

Elevation (nasal border)

No major vessel obscurity

3 Moderate Degree of Edema

Obstruction of $\geq 1$ segment of major blood vessels leaving disc ${ }^{\mathrm{a}}$

Circumferential halo

Elevation (all borders)

Halo (irregular outer fringe with finger-like extensions)

4 Marked Degree of Edema Total obscuration on the disc of a segment of a major blood vessel on the disc $^{\mathrm{a}}$

Elevation (whole nerve head, including cup)

Border obscuration (complete)

Halo (complete)

5 Severe Degree of Edema Obscuration of all vessels on the disc and leaving the disc ${ }^{\mathrm{a}}$

${ }^{a}$ Key features (major findings) for each grade 


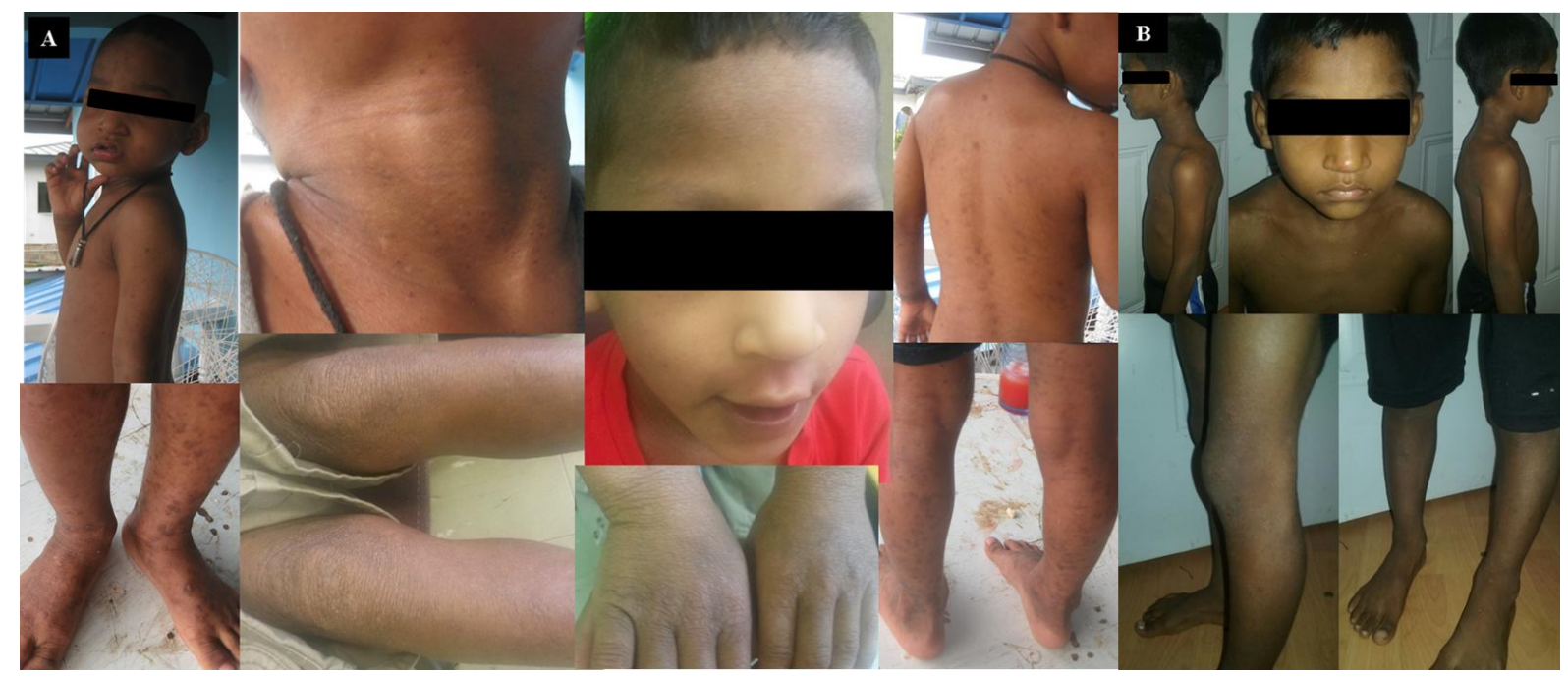

Fig 1. A. Severe rebound erythrodermic eczema following chronic systemic steroid. B. Resolution of eczema with oral azathioprine $2-3 \mathrm{mg} / \mathrm{kg}$, intensive moisturisers, dry wrapping and topical pimecrolimus.

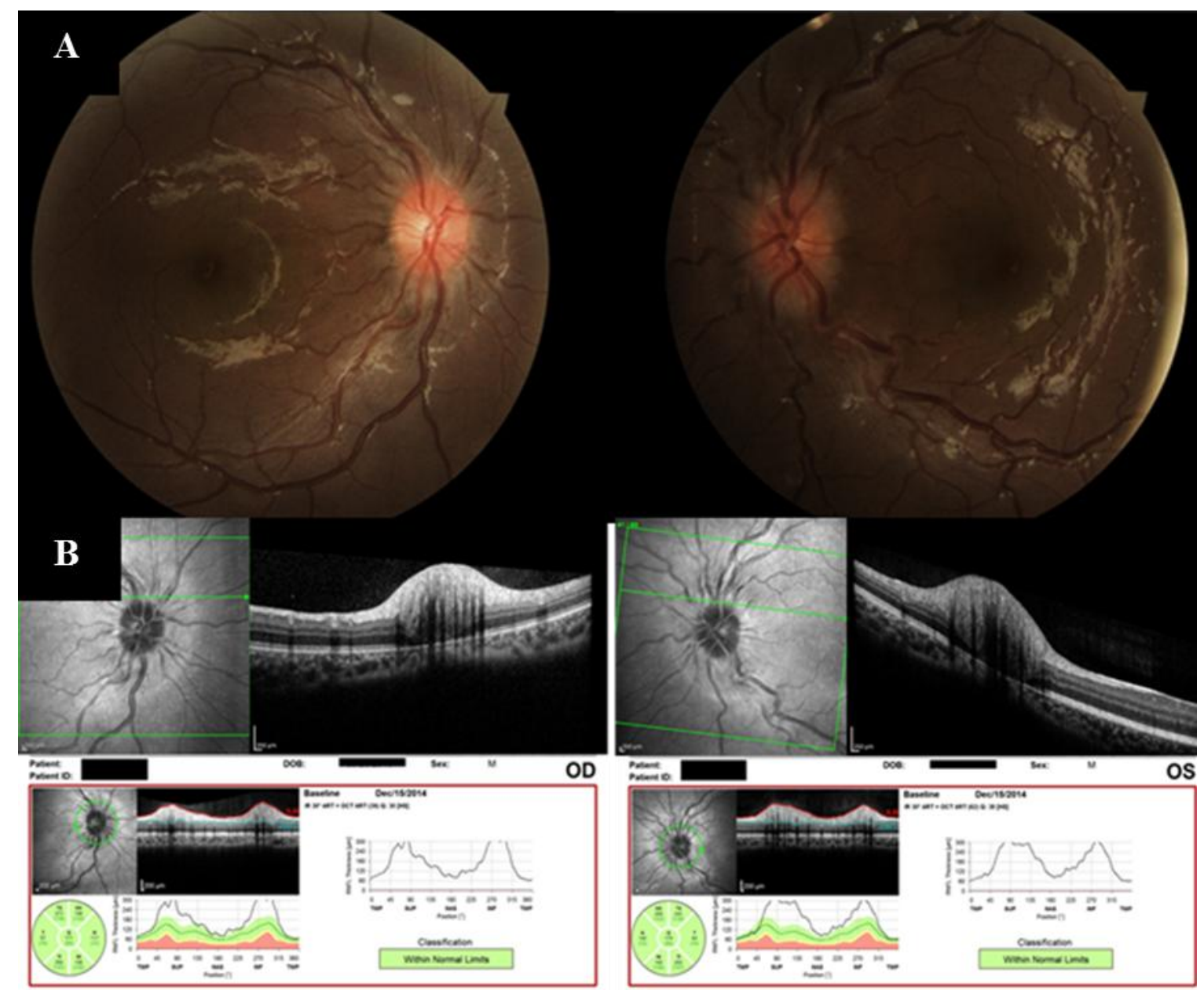

Fig 2. A. Colour stereoscopic fundus photographs of papilledema on presentation: RE Frisén Grade 1; LE Grade 2.

B. Enhanced depth imaging and retinal nerve fibre layer demonstrating optic nerve swelling on presentation. 


\section{Corticosteroid Induced Secondary Pseudotumor Cerebri Syndrome}

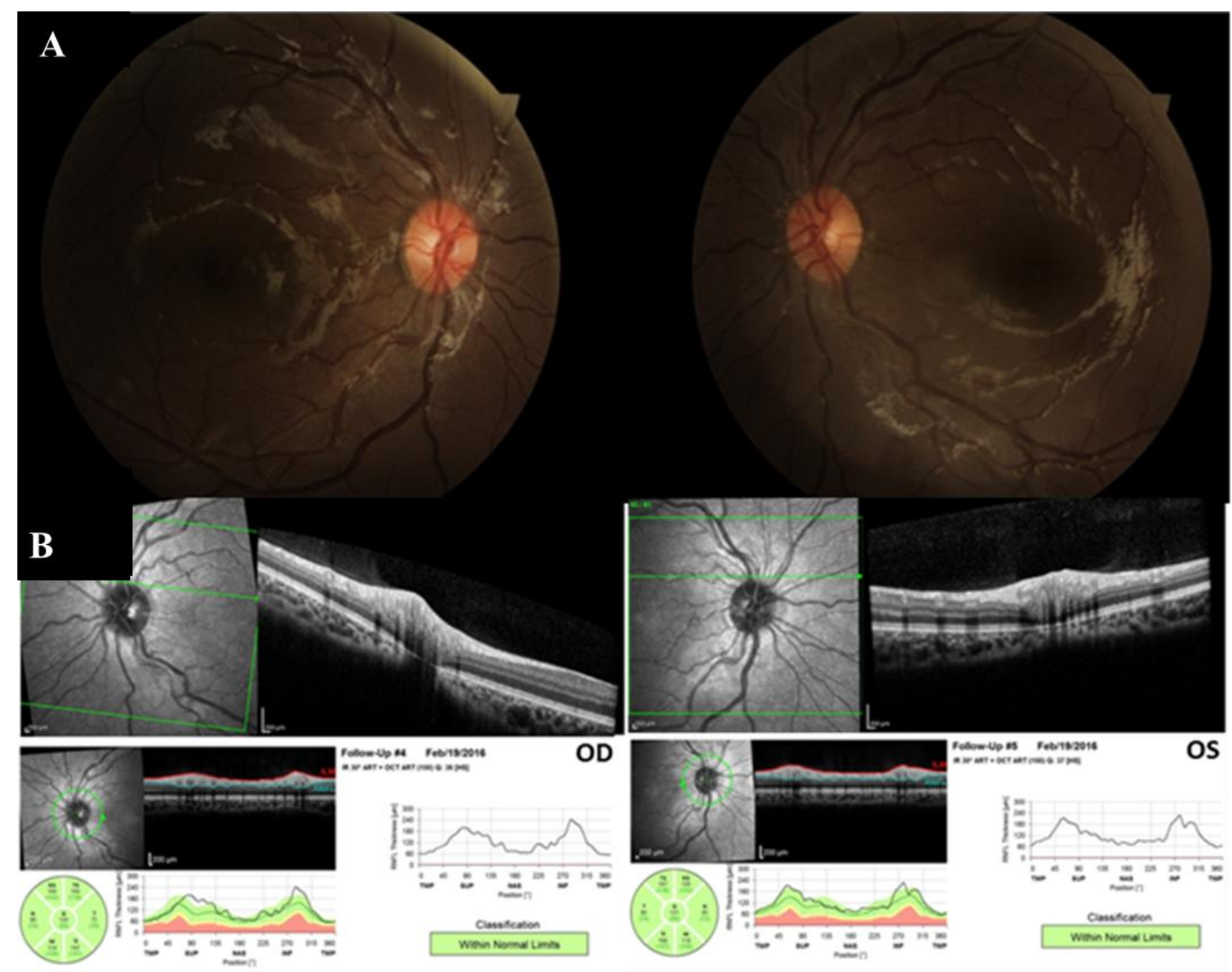

Fig 3. A. Colour stereoscopic fundus photographs. B. Enhanced depth imaging and retinal nerve fibre layer one year later on oral acetazolamide demonstrating resolution of papilledema. 


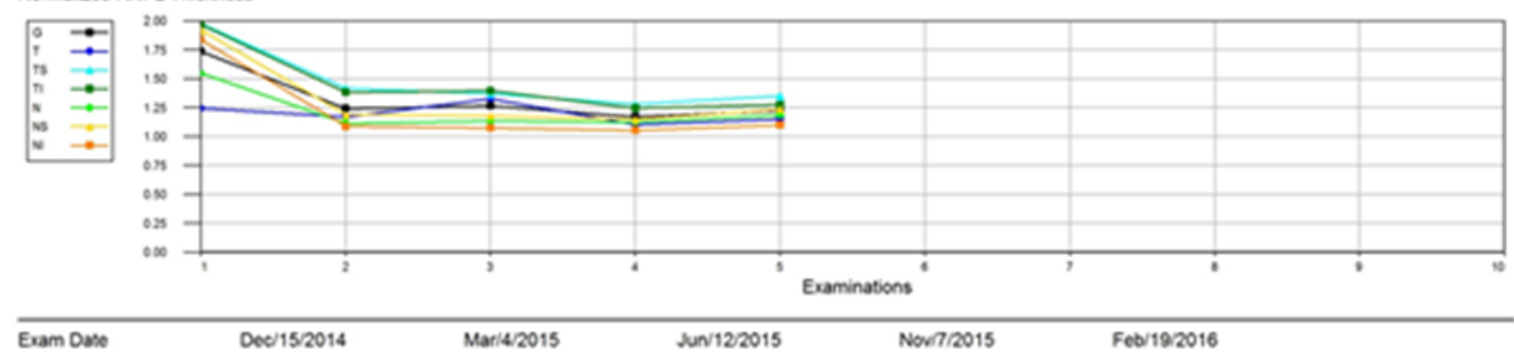

\section{Exam Date}

RNFL Trend Report
SPECTRALISS Tracking Laser Tomography

Patient:

Patient io:

$\infty$ :

Sex: M

OD

Normalized RNFL Thickness

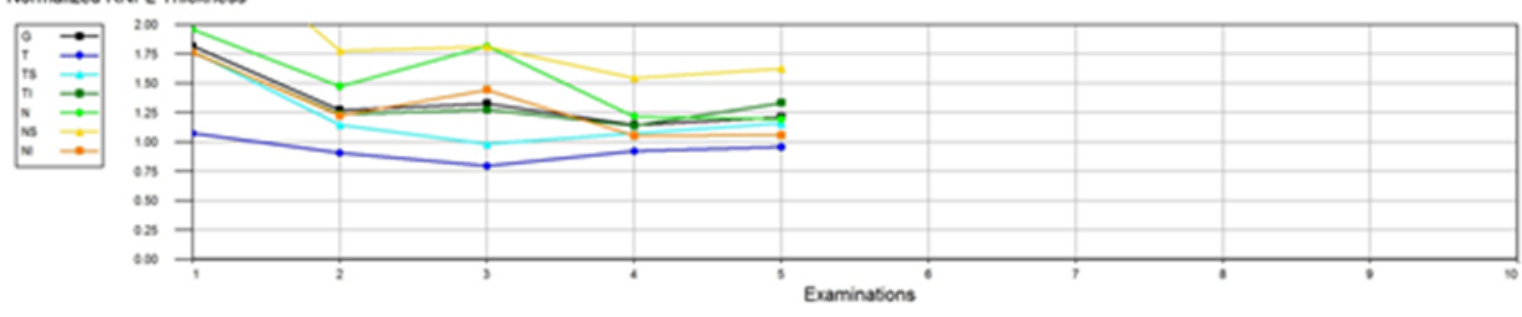

Exam Date

Dec/15/2014

Mar/4/2015

Jun/12/2015

Now7/2015

$\mathrm{Feb} / 19 / 2016$

Fig 4. Optical Coherence Tomography Trend Report during the clinical course documenting objective reduction/resolution of the papilledema in both eyes. 